\title{
A Study of Complete Kit Oriented Aerospace Product Manufacturing Model \\ Wei-Guo WANG
}

Beijing Institute of Aerospace Information Beijing, 100854, China

wangvito@qq.com

Keywords: kit-manufacturing, production control, product, batch, production cycle.

\begin{abstract}
Theory model of Kit-Manufacturing is established to improve the efficiency of aerospace product manufacturing. The definition and algorithmic method of Key Routing and Standard Production Batch is provided. The Kit-Manufacturing coefficient for monitoring product manufacturing process is defined and an evaluation method for Kit-Manufacturing is provided. The precondition of achieving Kit-Manufacturing is introduced.
\end{abstract}

\section{Introduction}

In the manufacturing process of aerospace product, the feature of Multi-variety, small batch and changing $\mathrm{BOM}$ is marked. Because of the complexity of manufacturing process, the problems of overstocked finished parts, delivery delay and higher cost is prominent [1]. In order to reduce the finished parts stock and production cycle, a complete kit controlling model of the entire manufacturing process of aerospace is introduced.

In the model of traditional production control, which kit is generally limited to the kit of assembly cycle, different kind of parts is always uncompleted in assembling cycle, then production plan is adjusted. Kit-Manufacturing[2], which is supplement of MRPII [3,4] and turns 'kit oriented production control' into 'kit based production control', request the kit of parts is considered during job plan is being made and executed. Kit manufacturing is proposed to improve the available production management theories. Wang summed up concept of related papers, and summarized that dispatch study of oriented to kit manufacturing is still insufficiency [5].

Kit manufacturing refers to the dynamic kit during the process of production by controlling the production temps of all kinds of parts, to make all kinds of parts to start and accomplish manufacturing at the same time by scheduling the different temps of parts $[6,7]$.

\section{Kit Manufacturing Model}

Kit manufacturing model is mainly composed of key routing, standard production batch, kit coefficient and the evaluation method of kit manufacturing.

Key Routing and Standard Production Batch. To get real time kitting during the whole process of parts production, we split the plan quantity of their father part to get shorter production cycle.

In the production process of a general discrete manufacturing enterprise, manufacturing time only accounts for a small part of the whole production cycle. Besides the actual production time, we call the extra time preparation time of work center.

Construction and use of color / shades of gray:

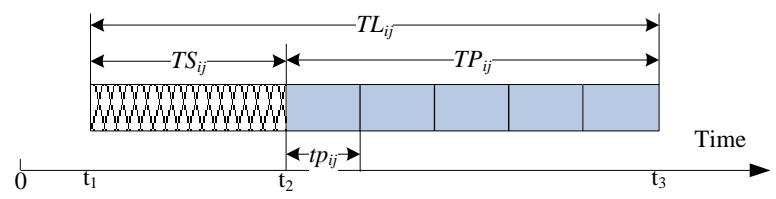

Fig.1 Preparation time and production time of a part 


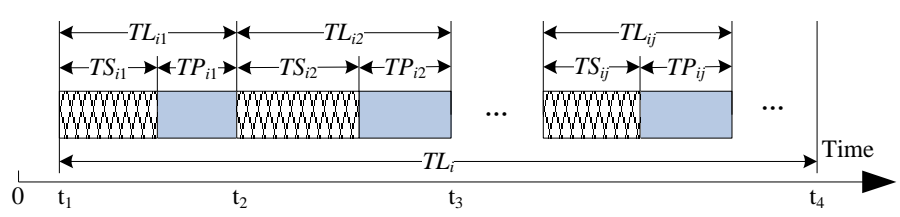

Fig.2 Processes in a production cycle

As in Fig.1, $t p_{i j}$ refers to the single production time of process $j$ of part $i$, and $T S_{i j}$ refers to the preparation time of the process. Set the batch quantity of part $i$ as $Q_{i}$, then $T P_{i j}=Q_{i} \cdot t p_{i j}$. As in Fig.2, the whole production cycle $T L_{i}$ of part $i$ is compared of several preparation time and production time, so that $T L_{i}=\sum_{j=0}^{J}\left(T S_{i j}+T P_{i j}\right)$. Several concepts are defined below before the kitting model is imported.

Economic batch quantity $Q^{E}$ : Economic batch quantity refers to the production batch with the minimum cost. Generally, economic batch quantity is integer times of feed quantity, carrying quantity and assembly quantity.

Production batch $Q^{L}$ : Production batch quantity is generally $1 / N$ of plan quantity.

Production period of part $i$ in planed quantity: $T L_{i}=Q_{i}^{P} \cdot t p_{i}+T S_{i}$

$N_{i}^{L}$ refers to the number of production batch: $N_{i}^{L}=Q_{i}^{P} / Q_{i}^{L}$

Production cycle of economic batch of part $i: T L_{i}^{L}=Q_{i}^{L} \cdot t p_{i}+T S_{i}$

Key routing: Key routing of a product refers to the longest routing of all parts. Set the planed quantity of component $u^{\prime}$, the key routing is calculated as following:

Set variant; Set component is assembled by parts, Set=0, go to Step2, and traverse all parts;

If, then go to 4HStep3, or go to HStep6;

Set the assembly quantity of part $\mathrm{i}$ as, and process number as J. Set $\mathrm{j}=0$, go to Step 5 and traverse all $\mathrm{J}$ processes.

If, go to HStep5, or and go to28H Step2;

Set production time of process $j$ of part $i$ as, preparation time as, then the whole production cycle of part I can be summarized as, if, then, set and go to9H Step4;

is the key routing of part when plan quantity as.

Standard production refers to the kit manufacturing mode, where all parts are manufactured in appropriate quantity, to make all parts being dynamic kit.

In JIT production mode, planed completion time of all parts should be at the same time. If planed completion time is $t_{K}$, the standard production batch can be calculated as follow.

Step1.Set the key routing of component as TLS, planed production quantity as. Set component is assembled by parts and $=0$, then go to Step 2 and traverse all I parts.

Step2.If, go to Step3, or go to Step4;

Step3. Set economic production batch of part $\mathrm{i}$ as. The production cycle could be obtained as and the number of production batch could be obtained as. If, then set, $\mathrm{i}=\mathrm{i}+1$ and go to 3HStep2;

Step4.Get the number of standard production batch of component as:

Step5.If, then go to33 Step6, or go to Step9;

Step6.The following steps should be done of part i:

a)Standard number of production batch $N_{i}^{L S}=\min \left(N_{i}^{L}, N^{L S}\right)$,

b)Standard production batch $Q_{i}^{L}=Q_{i}^{P} / N_{i}^{L S}$,

c) $k=0$ and go to Step2;

Step2.If $k<N_{i}^{L S}$, then go to or set $i=i+1$ and go to

Step3.For the $k$-th production cycle of part $I$, related parameters of plan production cycle could be obtained. 
a)Plan production cycle as $T L_{i}^{L S}=Q_{i}^{L} \cdot t p_{i}+T S_{i}=\left(Q_{i}^{P} / N_{i}^{L S}\right) \cdot t p_{i}+T S_{i}$,

b)Planed completion time as $t^{K}-\left(N_{i}^{L S}-k-1\right) \cdot T L S / N_{i}^{L S}$,

c)Planed begin time as $t^{K}-\left(N_{i}^{L S}-k-1\right) \cdot T L S / N_{i}^{L S}-T L_{i}^{L S}$,

d)Set $k=k+1$ and go to Step2.

The result is evaluated.

Kit coefficient. The quantity of parts could be assembled of a component is up to the minimum quantity of completed parts. For example, component $\mathbf{p}$ is composed of $x_{1}, x_{3}, x_{4}$ and $x_{5}$, which quantity is $\mathbf{2 , 4 , 6 , 8}$ and 10 . The completion quantity of $p$ at the moment of $\boldsymbol{t}$ can be expressed as $Q_{p}^{F}(t)=\left\{Q_{1}^{F}, Q_{2}^{F}, Q_{3}^{F}, Q_{4}^{F}, Q_{5}^{F}\right\}$.

Tab.1 Quantity of parts of component $\mathrm{p}$ at different moment

\begin{tabular}{lll}
\hline Moment & completed quantity & Surplus of Assembling \\
\hline$t_{1}$ & $\{2,0,0,0,0\}$ & $\{2,0,0,0,0\}$ \\
$t_{2}$ & $\{2,4,6,8,0\}$ & $\{2,4,6,8,0\}$ \\
$t_{3}$ & $\{4,8,12,16,20\}$ & $\{0,0,0,0,0\}$ \\
$t_{4}$ & $\{5,8,12,16,20\}$ & $\{1,0,0,0,0\}$ \\
\hline
\end{tabular}

Completion rate of part $i: F_{i}=\left(Q^{F} / Q^{P}\right) \times 100 \%$, where $Q_{i}^{F}$ is the completion quantity of part $i$. The maximum completion rate can be expressed as $F^{\max }=\max _{i}\left\{F_{i}\right\}$. And the kit coefficient of part $i$ is $S_{i}=F_{i} / F^{\max }$. The completion rate and kit coefficient of component $\mathrm{p}$ at different moment is listed in Tab.2.

Tab.2 The completion rate and kit coefficient of component $\mathrm{p}$ at different moment

\begin{tabular}{ccccc}
\hline Time & Plan quantity & completed quantity & completed rate & Kitting coefficient \\
\hline$t_{1}$ & $\{2,0,0,0,0\}$ & $\{0.2,0,0,0,0\}$ & $\{2,0,0,0,0\}$ \\
$t_{2}$ & $\{2,4,6,8,0\}$ & $\{0.2,0.2,0.2,0.2,0\}$ & $\{1,1,1,1,0\}$ \\
$t_{3}$ & $\{10,20,30,40,50\}$ & $\{1,2,3,4,5\}$ & $\{0.1,0.1,0.1,0.1,0.1\}$ & $\{1,1,1,1,1\}$ \\
$t_{4}$ & $\{4,8,12,16,20\}$ & $\{0.4,0.4,0.4,0.4,0.4\}$ & $\{1,1,1,1,1\}$ \\
$t_{5}$ & $\{5,8,12,16,20\}$ & $\{0.5,0.4,0.4,0.4,0.4\}$ & $\{1,0.8,0.8,0.8,0.8\}$ \\
\hline
\end{tabular}

\section{Evaluation of Kit Manufacturing}

The target state of kit manufacturing is that completion rate of every part keeps pace which each other and kit coefficient is equal to 1 .

Set $u^{\prime}$ as parent component of part $i$, then completion rate of $u^{\prime}$ is $F_{u^{\prime}}^{\prime}=\min _{i}\left\{F_{i}\right\}$ and kit coefficient of $u^{\prime}$ is $S_{u^{\prime}}^{\prime}=\min _{i}\left\{S_{i}\right\}$. Set $q_{i}^{c}$ as the assembly quantity of part $i$ in $u^{\prime}$, then $Q_{u^{\prime}}^{F}=\min _{i}\left(Q_{i}^{F} / q_{i}^{c}\right)$.

Completion rate of a component, which is defined as the weighted average completion rate of all 
parts, is $F=\sum_{i=0}^{I} T R_{i} \cdot F_{i} / \sum_{i=0}^{I} T R_{i}$. And kit coefficient of a component is defined as the weighted average kit coefficient of all parts, is $S=\sum_{i=0}^{I} T R_{i} \cdot S_{i} / \sum_{i=0}^{I} T R_{i}$. Set the start time and completion time of $k$-th production cycle of part $i$ as $t_{i}^{k}$ and $t_{i}^{k+1}$. Set completion rate of part $i$ as $F_{i}(t x)=\left\{\begin{array}{cc}0, & t^{k} \leq t x<t_{i}^{k} \\ \left(t x-t_{i}^{k}\right) / T L_{i}^{L S}, & t_{i}^{k} \leq t x<t_{i}^{k+1} \\ 1, & t_{i}^{k+1} \leq t x \leq t^{k+1}\end{array}\right.$, then the average kit coefficient of part $i$ is $\overline{S_{i}^{k}}=\frac{1}{T L^{L S}} \int_{t^{k}}^{t^{k+1}} \frac{F_{i}(t x)}{F^{\max }(t x)} d t x$. The completion curve of different parts is different, so $F^{\max }(t x)$ equals to different $F^{\max }(t x)$ curve in different domain of $t x$. As in Fig.3b, the kit coefficient of Part2 could be obtained as:

$$
S_{2}(t x)=\left\{\begin{array}{cc}
\frac{\left(k / N^{L S}\right)}{(x / 100)}=\frac{100 k}{N^{L S} \cdot t x}, & 100 k<N^{L S} \cdot t x \leq 100 k+20 \\
-\frac{200 k+100}{3 N^{L S} \cdot t x}+\frac{5}{3}, & 100 k+20<N^{L S} \cdot t x \leq 100 k+50 \\
\frac{(x-40 k-20) / 60 N^{L S}}{(x-40 k-20) / 60 N^{L S}}=1, & 100 k+50<N^{L S} \cdot t x \leq 100 k+80 \\
1 / 1=1, & 100 k+50<N^{L S} \cdot t x \leq 100 k+80
\end{array}\right.
$$

$S_{0}(t x)$ and $S_{1}(t x)$ could be obtained by the same method.

The average kit coefficient of part $i$ could be obtained as

$$
\overline{S_{i}}=\frac{1}{T L S} \cdot \sum_{k=0}^{N^{L S}}\left(\overline{S_{i}^{k}} \cdot T L^{L S}\right)=\frac{1}{T L S} \cdot \sum_{k=0}^{N^{L S}}\left(\frac{\int_{t^{k}}^{t^{k+1}} S_{i}(t x) d t x}{T L S / N^{L S}} \cdot T L S / N^{L S}\right)=\frac{1}{N^{L S}} \cdot \sum_{k=0}^{N^{L S}}\left(\frac{\int_{t^{k}}^{t^{k+1}} S(t x) d t x}{T L S / N^{L S}}\right) .
$$

So that $\overline{S_{2}}=\frac{1}{N^{L S}} \cdot \sum_{k=0}^{N^{L S}}\left[1-\left(k \ln \frac{100 k}{100 k+20}+\frac{2 k+1}{3} \ln \frac{100 k+50}{100 k+20}\right)\right]$.

Since $f(k)=1-\left(k \ln \frac{100 k}{100 k+20}+\frac{2 k+1}{3} \ln \frac{100 k+50}{100 k+20}\right)$ is an increasing function, so that the average kit coefficient $\overline{S_{i}^{k}}$ is also an increasing function of $k$. And kit coefficient $\overline{S_{2}}$ could be increased with the increase of $N^{L S}$. 


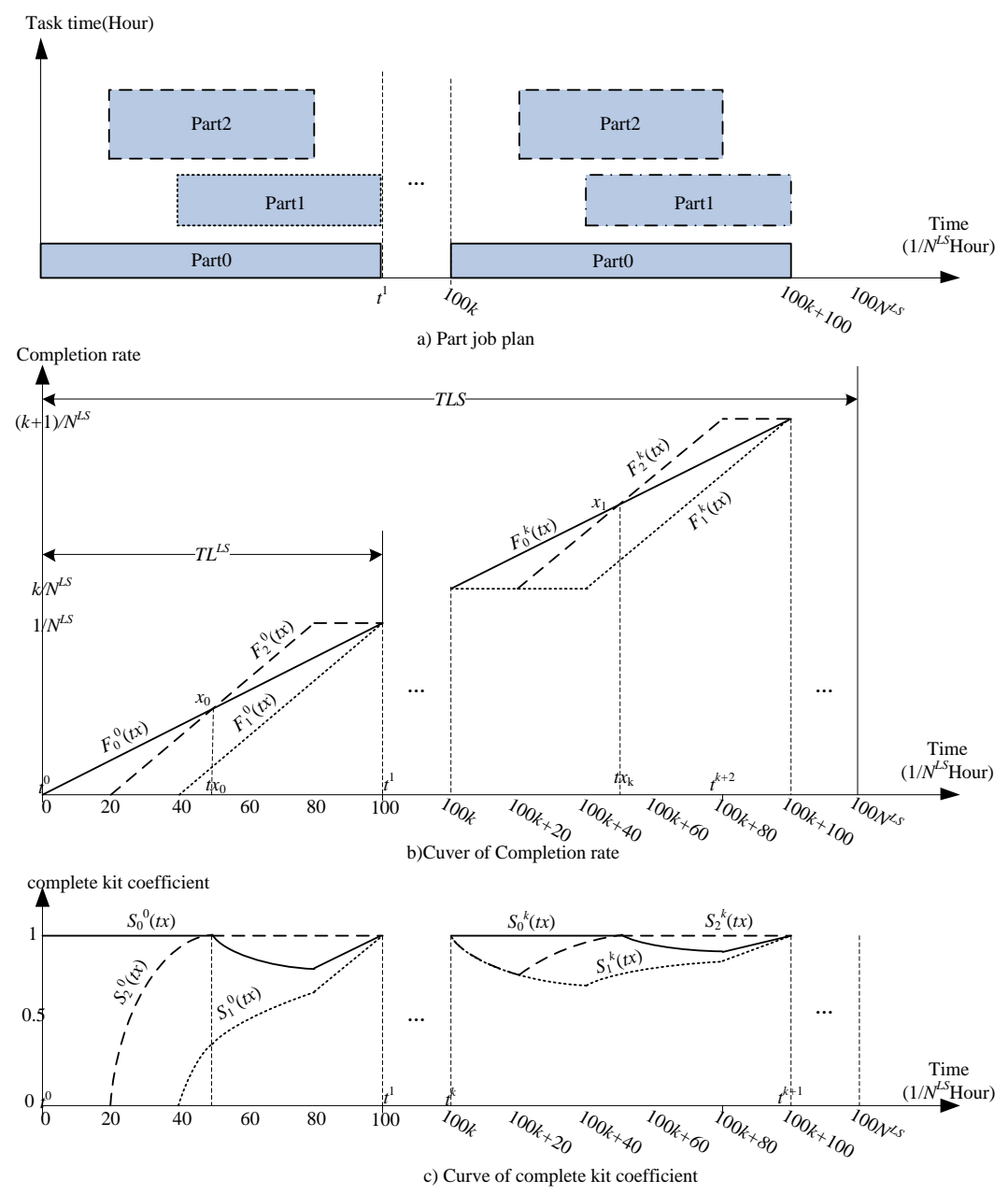

Fig.3 Diagram of kit manufacturing evaluation.

In kit manufacturing mode, methods such as standard production batch calculation and monitoring of kit coefficient should be used to optimize the whole production process.

\section{References}

[1] M. Stevenson, L. C. Hendry, Aggregate load-oriented workload control: A review and a reclassification of a key approach, International Journal of Production Economics, Vol.104, No. 2, 2006, pp. 676-693.

[2] B. Ronen, The complete kit concept, International Journal of Production Research, Vol.30 No.10, 1992, pp.2457-66.

[3] J. Q. Gou, D. Chang, ERP Principles and Practices, (Tsinghua University Press, Beijing, 2005), pp.5-6.

[4] C. Kong, MRPII/ERP Principles and Applications, (Tsinghua University Press, Beijing, 2006), pp. $32-36$

[5] L. P. Wang, Production Scheduling of Discrete Manufacturing under Complete Kit Concept, PhD thesis, Dalian University of Technology (Dalian, 2009).

[6] X. P. Li, X. X. Ning, The algorithm of dynamic completeness of parts based on line-unit management in assembly process, Computer integrated manufacturing systems, Vol.5, No.4, 1999, pp.66-71.

[7] Developing trend of multi product and small batch manufacturing technology and management, Journal of Xi' an Institute of Technology, Vol.17, No.3, 1997, pp. 242-247 